\title{
Malformaciones de la pared torácica en niños
}

\author{
Dres. Oscar Santos, ${ }^{\prime}$ Oscar Gómez, ${ }^{2}$ Claudio Arret., ${ }^{2}$ Luis León, ${ }^{2}$ Helmut Jaeger,
}

\section{Chest wall deformities in children}

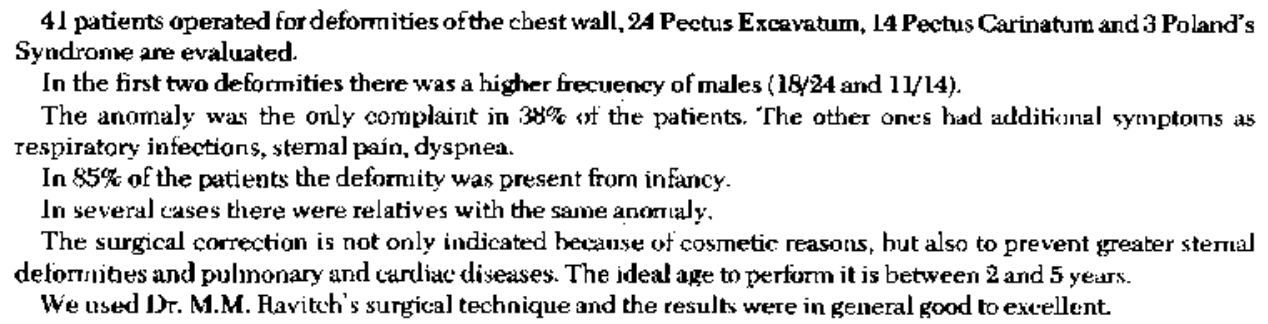

Se analizan las historias clinicas de los pacientes operados por malformaciones de la pared torácica, desde 1970 hasta 1981, en el Centro Cardiovascular del Hospital Luis Calvo Mackenna.

El total de níños operados es de 41 y se divide en 3 grupos:

- Pectus Excavatum 24 pacientes

- Pectus Carinatum 14 "

- Síndrome de Poland 3 "

Siete de ellos fueron enviados de provincias.

\section{PECTUS EXCAVATUM}

Constituye la patologia más frecuente en nuestra serie con el 59\% de los casos. (Fotos 1 y 2 ).

Corresponde a una depresión del estemón distal, simétrica o asimétrica, con o sin prominencia de los cartílagos costales y que puede tener distintos grados, dependiendo de la superficie $y$ de la profundidad que alcance. ${ }^{1,2,3}$

Fisiopatologia Estudios realizados por diferentes autores han demostrado que esta anomnalidad

\footnotetext{
I Becado Cirugia Infantil S.N.S.

${ }^{2}$ Centro Cardiovascular-Hospital Calwo Mackerma. Estuela de Medicina-Universidad de Chile-Secle Oriente.
}

está asuciada a varias alteraciones funcionales siendo importante una: reducción del débito y volumen de eyeccion cardiaca de hasta un $40 \%$ en reposo, con incapacidad de aumentar durante el ejercicio, lo que ha sido demostrado a través de cateterización del corazón. ${ }^{.5}$ Un $95 \%$ de los pacientes presentan soplo holosistólico suave. ${ }^{2}{ }^{\hat{6}}$

Hay alteraciones electrocardiográticas que en un buen porcentaje desaparecen después de la operación. ${ }^{3}$ Estudios realizados con radioisótopos demuestran alteraciones pulmonures tanto de la perfusión como de la ventilación, que se modifican significativamente después de la corrección. ${ }^{3}$

Por lo tanto la operación no sólo es cosmética sino cue realizada oportunamente, previene mayores deformidades de la columna y anomalías cardiopulmonares.

La edad ideal para la operación es entre los 2 y 5 años."

Sexo: 18 de nuestros pacientes son hombres $(75 \%)$ y 6 son mujeres. Otras publicaciones dan cifras parecidas. ${ }^{3,4}$

Mativo de consulta: La deformidad es evidente en todos los pacientes y constituye por sí sola, la única manifestación de 9 de $\operatorname{los} 24$ pacientes (38\%). El62\% restante (15) presentaban sintomatología agregada que se desglosa así: 


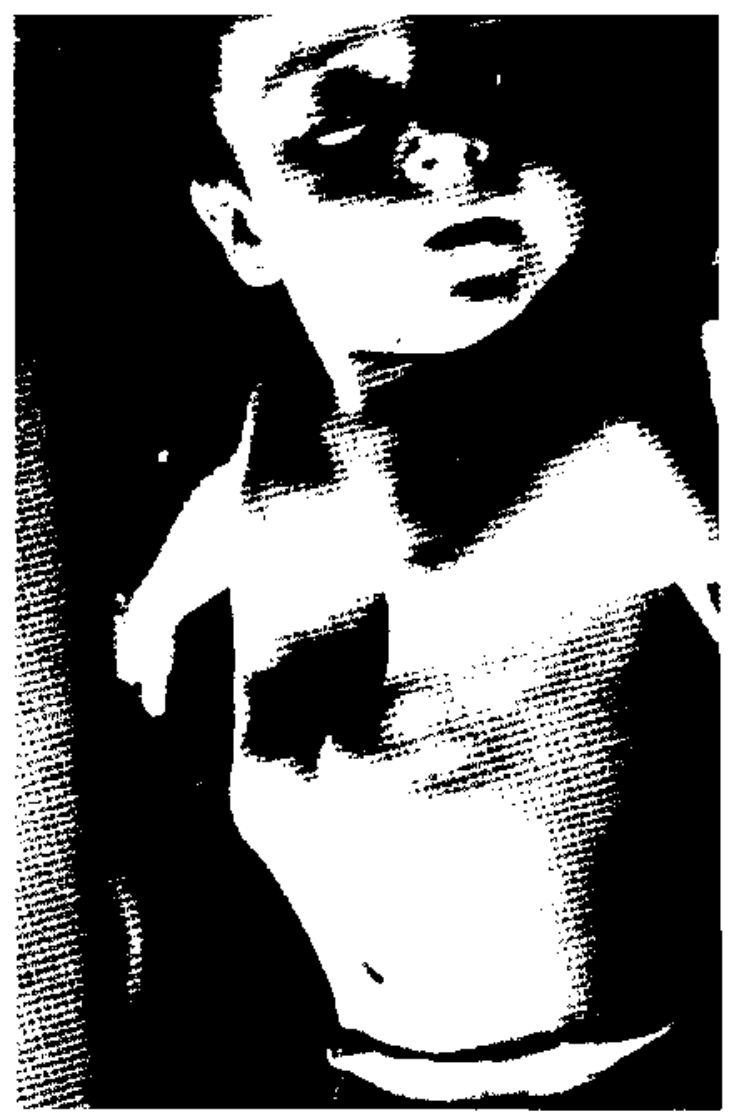

Foto 1. Pectis excavatum estado preoperatorio.

Infecciones respiratorias a repetición (Bronquitis, bronconeumonía, atelectasia) 7 Dolor estemal o sensación de opresion 6

Disnea

Estridor 3 3

En 21 pacientes ( $88 \%$ ), se notó la deformidad antes de los 3 años de edad, presentándose en la niayoría de los casos desde el nacimiento. En el resto la aparición es tardía y corresponden a 2 casos temeninos cuya deformidad apareció alrededor de los 12 años y a un caso masculino, a los 7 añes.

Antecedentes familianes. En las tichas se consigna que 4 de los pacientes tenían uno o más tamiliares cercanos con igual deformidad en grado leve. Debemos dejar establecido que este antecedente no se obtuvo en todos los casos por lo que su incidencia pudiera ser mayor.

Operacion. El tiempo transcurrido entre la primera consulta en el Servicio y la operación fue de 1 mes a 3 años. Nueve de los pacientes fueron operados antes de los 2 meses y corresponden a aquellos

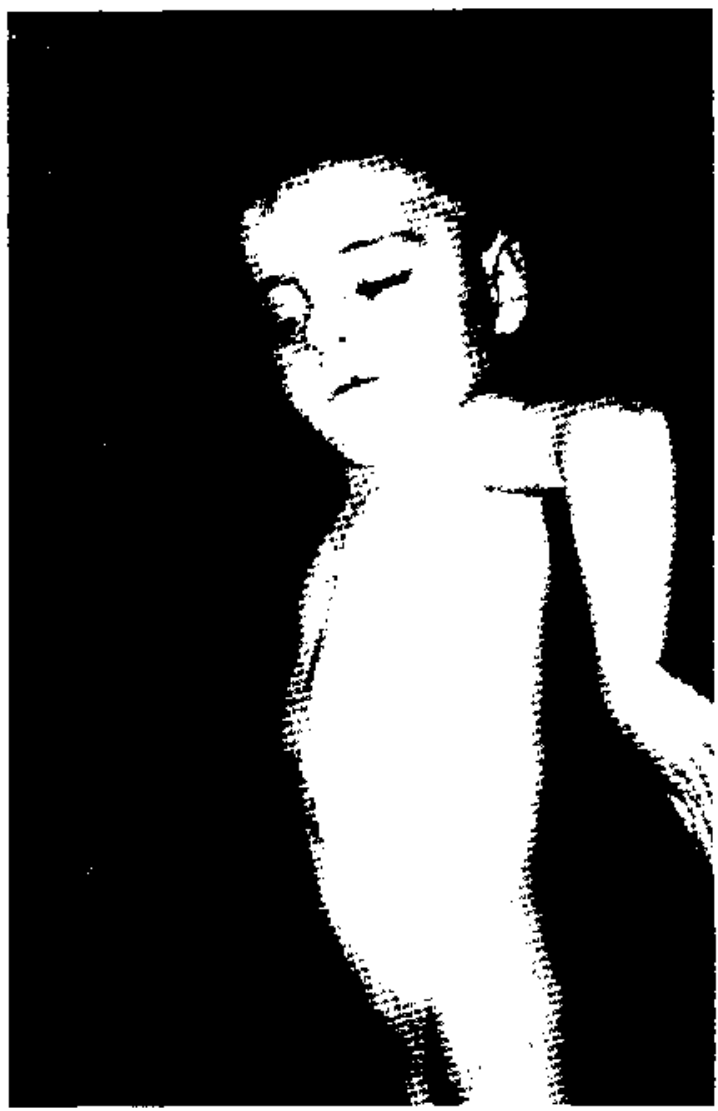

Foto 2. Pectus excavahum estado postoperatorio.

con deformidades graves. En el resto se observo sı evolución, se hizo Kinesiterapia y fuerom operados porque la deformidad iba progresando o los síntomas eran más intensos.

La edad en que los pacientes se operaron varió entre 6 meses y 15 años, con un promedio de casi 7 años.

Técnica quirúrgica. En sólo 1 paciente se usó la técnica de Rebhein con colucación de 2 férulas metálicas. ${ }^{\text {I0 }}$

En el resto de los pacientes se usó la técnica descrita por M.M Ravitch, ${ }^{1,3}$ que consiste desinsertar del estenón ambos músculos pectorales y los rectos anteriores, para exponer ampliamente los cartílagos costales y costillas comprometidas los que se resecan dejando "in situ" el pericondrio y periosteo. Luego se hace una osteotomía transversa estemal en cuña, por escima de la depresión, la que se lija con puntos elevindo así la zona hundida. El par de cartilagos inmediatamente inferior a la cuña no se reseca, sino que se corta en bisel y se traslapan sus bordes fijándolos con 
puntos para aumentar la estabilidad del cuerpo estemal. En la primera mitad de la casuística se hizo plicatura de los lechos pericondriales, to que posteriormente se estimo innecesario. En los últimos pacientes se han desinsertado del esternón deprimiendo todas las inserciones musculares intercostales y pericondriales, dejándolo completamente libre. Finalmente se reinsertan en el esternón los músculos pectorales y rectos anteriores $y$ se dejan drenajes aspirativos. No se colocan elementos externos de fijación.

La incisión más frecuente tue la media vertical en 18 casos. En 6 pacientes se usó la arciforme submamaria por razones estéticas, $y$ es la vía preferida actualmente.

Se resecaron 5 pares de cartílagos en 4 casos, 4 pares en 15 casos y 3 pares en 4 casos, lo que da una idea de la magnitud de la lesión.

Complicaciones inmediatas: No hubo mortalidad.

En 7 pacientes se abrió pleura por lo que fue necesario dejar drenaje bajo agua, lo que evolucionó sin problemas. En $\mathbf{5}$ hubo algún grado de

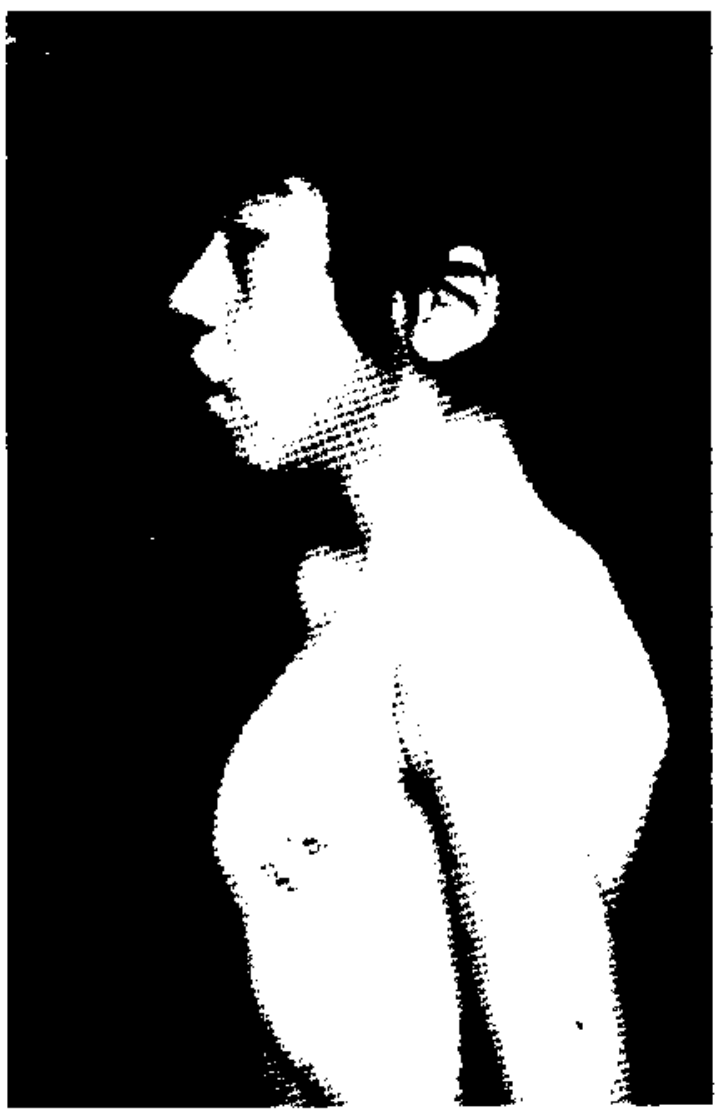

Foto 3. Pectus carinatum estado preoperatorio. infección y dehiscencia de suturas a pesar de que a todos se dejó tratamiento antibiótico profiláctico. Esta eventualidad casi ha desaparecido al mejorar el sistema de drenaje subcutáneo.

Evolución. Los pacientes permanecieron entre $3 y$ 42 dias hospitalizados después de la operación, con un promedio de 14 días. Los controles postoperatorios mostraron que la corrección fue satisfuctoria en 20 de los casos $(83 \%)$. Sólo un paciente presentó reproducción total del Pectus, fue reoperado, persistiendo el mal resultado, to que se atribuye a que presentó infecoción de consideración. En otros 3 hubo reproducción parcial y también están asociados a infección. La edad también parece influir, siendo menos satisfactorios los resultados en los nin̄os mayores al igual que en el único menor de 2 años operado.

\section{Pectus Carinatum.}

Es la segunda patología en importancia con el $34 \%$ de los pacientes ( 14 casos) (Fotos 3 y 4 ).

Corresponde a un solevantamiento del esternón con aumento del diảmetro torácico antero-

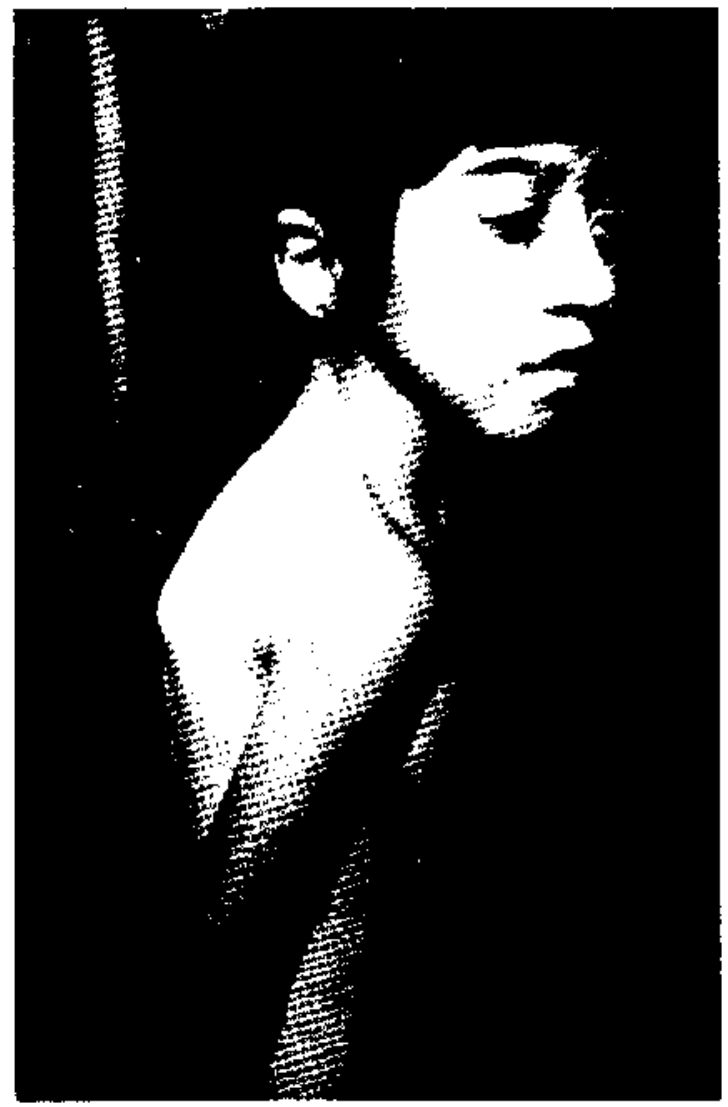

Foto 4. Pectus carinatum estado postoperatorio. 
posterior, que puede ser simétrico o asimétrico y de extensión variable. ${ }^{1,2,3}$

Se acompaña de alteraciones de la columna.

Sexo. 11 pacientes fueron hombres (78\%) y $3 \mathrm{mu}$ jeres. 3.4

Motivo de la consulto. A] igual que en el Pectus Excavatum hay pacientes que lo único que presentan es la deformidad, sin otra alteración, pero que los lleva a solicitar la operación lo que ocurrió en 5 de ellos (36\%).

Los restantes presentaban además algunas de las siguientes manifestaciones.

- Infecciones respiratorias

(Brorkquitis, bronconeumonías) 5

- Sensación de ahogo 4

- Dolor estemal

- Retardo desarrollo psicomotor 3

- Agenesia pectoral mayor unilateral. 1

La aparición de la deformidad fue notada antes de los 3 años en 11 de ellos (79\%). En 2 casos femeninos la deformidad habria comenzado a los 12 y 7 años respectivamente y en un caso masculino a los 12 años.

Antecedentes famificres. En 3 casos se consigna antecedente de familiar cercano que presenta igual deformidad en grado leve. ${ }^{4}$

Openación: Desde la primera consulta en el Servicio hasta la operación transcurrieron de 3 días a 2 años con un promedio de $61 / 2$ meses.

La edad en que se operaron varió de 2 a 18 años siendo el promedio de casi 9 años.

Técnica quirúrgica. En todos se utilizó la técnica de M.M. Ravitch ${ }^{1,7}$ que consiste en desinsertar ambos músculos pectorales mayores para resecar los cartílagos costales comprometidos, manteniendo los lechos pericondriales, en los que se hace una plicatura, con lo que se logra a veces, la corrección inmediata del esternón.

Otros casos requieren además, osteotomías simples transversas del estemón o longitudinales en los casos asimétricos. En seguida se reinsertan los pectorales para mantener la fijación dejando 2 drenajes aspirativos.

La incisión más frecuente fue la media vertical en 9 casos, y en 5 se hizo transversa submamarias, siendo ésta la preferida actualmente.

La resección de cartílagos varió de acuerdo a la deformidad y en 2 casos asimétricos la corrección fue unilateral. Se realizó osteotomía del estemón en la mitad de los pacientes.

Complicaciones inmediatas. En un paciente se abrió pleura requiriendo drenaje y uno presentó infec- ción de la herida con dehiscencia parcial de sutura, que evolucionaron bien.

Evolución. Los resultados son excelentes. En todos se consigna buen resultado estético con la operaciôn, disminuyendo el diámetro anteroposterior del tórax y quedando con buena elasticidad torácica.

Llama la atención y esto es válido para todos los pacientes que hay tendencia a la cicatriz queloidea, especialmente en la incisión media vertical pero que con el tiempo tiende a disminuir.

\section{Sindrome de Poland}

Es una malformación que se caracteriza por la ausencia unilateral de los cartilagos costales $2^{\circ}, 3^{\circ}$ y $4^{\circ}$, que se acompaña de agenesia del pectoral mayor ipsilateral y a veces de alteraciones del pulmón del mismo lado., ${ }^{2,3}$

En general es bien tolerada, pero puede haber compromiso respiratorio importante, por respiración paradojal. Además el pulmón subyacente está desprotegido frente a traumatismos.

Se operaron tres pacientes: Niña de 21 días que fue enviada pensando en cardiopatía congénita, y que había presentado dos episodios de bronconeumonía. Niña de 8 años que presentaba defecto costal desde el nacimiento con respiración paradojal. Nin̄o de 2 años con resfríos conitinuos y disnea de esfuerzo.

Operación. Está orientada sólo a reparar la ausencia de cartilagos costales, para lo cual se interponen injertos tomados sin periosteo de costillas inferiores y que se fijan con puntos. En un caso se cubrieron los injertos con malla de Dracon.

Evoiución. No hubo complicaciones operatorias. Los injertos prendieron bien, manteniéndose en su lugar, pero el resultado estético no es del todo satisfactorio por la agenesia del músculo pectoral. Sin embargo se cumplió con proteger el tejido pulmonar de traumatisinos externos y suprimir la respiración paradojal que son los objetivos principales de esta operación.

\section{RESUMEN}

Se revisan las historias clinicas de 41 pacientes operados por malformaciones de la pared toracica en el Hospital Luis Calvo Mackenna: 24 con Pectus Exeavatum, 14 con Pectus Carinatum y 3 con Síndrome de Poland. Hay una clara mayor incidencia en el sexo masculino en las dos primeras patologias (18/24 y $11 / 14$, respectivamente).

La deformidad es evidente y por si sola motivo 
de consulta en el 38\% de los pacientes; el resto presenta sintomatolugia agregada.

Dicha deformidad aparece precozmente en el $85 \%$ de los pacientes.

El antecedente de tamiliar cercano con igual deformidad es significativo.

La operación no sólo es cosmética sino que realizada oportunamente previenen mayores deformidades y anomalias cardiopulmonares evidentes.

La técnica quirúrgica utilizada es la de M.M de Ravitch y el resultado es altamente satisfactorio.

\section{REFERENCLAS}

${ }^{1}$ Ravitch, M M.: Congenital deformities of the chest wall and their sperative correction. Philiadelphia. W.B. Saunders Corpany, 1977

${ }^{2}$ Ravitch, M.M et of: Pediatric Surgery. Year Bouk Medinal Publisher, 1979.
${ }^{3}$ Holder, T.M. and Ashoruft. K.W.: Pediatric Surgery. Philadel phia, W.B. Saunders Company, 1980.

4 Gray, S.W. and Skandalakis, J.E: Embriology for Surgenns. Philadelphia. W.B. Sunnders Company.

${ }^{5}$ Beser. G.D. et al: Impaimment of cardiac function in patients witl Pectus Excenvatum, with improvement after cperative correction, N. England J. Med. 287-267,.1972.

${ }^{6}$ Solomon d. et of: Thoracte skeletal abmonnalities in jdiopatic mitral valve prolayse A.M. J. Cardiol 36: 32, 1975.

${ }^{7}$ Welch K.J. and Vos, A. Surgical correction of Pectus Carinatum J. Pediatric Surg. 3: 659, 1973.

${ }^{8}$ Comer, T.P. and Lymn, H.B. Indications for Surgical Treatment of Chest-Wall Defonnities in Children. Mayo Clinic Proc. 42: 13-22, 1967.

${ }^{9}$ Holcomb, G.W.: Surgical Cortection of Pectus Excavatum. J. Ped. Suns. 12: 295. 1977.

${ }^{10}$ Rehbein, F, and Wernicke, H.M. The operative treatuinent of the tumel chest. Anch. Dis. Child. 32: 5, 1957.

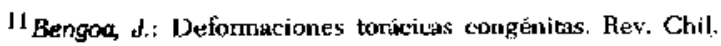
Ped. 35: 117-133, 1964 .

12 Lirc, E. Defornaciones de la pared torácica. Cuad. Chil. Cir. 20: 68-72, 1976.

${ }^{13}$ Cancino E y atros. Once casos de Pectos Carinatum. Cuad. Chil. Cir. 20: 7:3-78, 1976. 